\title{
BMJ Open Computerised memory specificity training (c-MeST) for the treatment of major depression: a study protocol for a randomised controlled trial
}

\author{
David J Hallford, ${ }^{1}$ David W Austin, ${ }^{1}$ Filip Raes, ${ }^{2}$ Keisuke Takano ${ }^{3}$
}

To cite: Hallford DJ, Austin DW, Raes F, et al. Computerised memory specificity training (c-MeST) for the treatment of major depression: a study protocol for a randomised controlled trial. BMJ Open 2019;9:e024508. doi:10.1136/ bmjopen-2018-024508

- Prepublication history for this paper is available online. To view these files, please visit the journal online (http://dx.doi. org/10.1136/bmjopen-2018024508).

Received 30 May 2018

Revised 30 January 2019

Accepted 31 January 2019

Check for updates

(c) Author(s) (or their employer(s)) 2019. Re-use permitted under CC BY-NC. No commercial re-use. See rights and permissions. Published by BMJ.

${ }^{1}$ School of Psychology, Faculty of Health, Deakin University, Burwood, Victoria, Australia ${ }^{2}$ Faculty of Psychology and Educational Sciences, KU Leuven, Leuven, Vlaanderen, Belgium

${ }^{3}$ Division of Clinical Psychology and Psychotherapy, Department of Psychology, Ludwig-

Maximilians-University Munich, Munich, Bavaria, Germany

Correspondence to

Dr David J Hallford;

david.hallford@deakin.edu.au

\section{ABSTRACT}

Introduction Major depression is a prevalent and debilitating disorder, but many sufferers do not receive support or respond to current treatments. The development of easily accessible and low-intensity treatments that have clear cognitive mechanisms of change is indicated. Memory specificity training (MeST) is an intervention for depression that targets deficits in recalling detailed memories of past experiences through repeated practice of autobiographical memory retrieval. This randomised controlled trial will assess the efficacy of an online, computerised version of MeST (c-MeST).

Methods and analysis Adults aged 18 and over with a current major depressive episode (MDE) will be recruited and randomised to have access to the seven session, online c-MeST programme for 2 weeks, or to a wait-list control group. The primary outcomes will be diagnostic status of MDE and self-reported depressive symptoms at postintervention. One-month and three-month follow-ups will be collected. Increases in autobiographical memory specificity will be assessed as a mediator of change, as well as other variables thought to contribute to reduced memory specificity, such as rumination and cognitive avoidance.

Ethics and dissemination Ethics approval has been granted by the Deakin University Human Research Ethics Committee to conduct the study (ID: 2017_168). The findings will be disseminated through scholarly publications and workshops and will inform future trials, such as with an active comparator or as an adjunct treatment.

Trial registration number ACTRN12618000257268; Preresults.

\section{INTRODUCTION}

Major depression is a prevalent and debilitating mental health disorder. More than 300 million people are currently living with depression worldwide, and it is the leading cause of ill health and disability. ${ }^{1}$ Currently, there exists a number of evidence-based psychosocial and pharmacological treatments. However, a range of barriers exist to the provision of effective treatments, including low help-seeking rates, ${ }^{2}$ difficulties

\section{Strengths and limitations of the study}

This trial will determine the efficacy of an online, low-intensity adaptation of an intervention.

- The proposed mechanisms of change will be assessed.

- The study will not include an active comparator group.

The study will not use a long-term follow-up.

with access ${ }^{3}$ and the fact that many individuals simply do not respond to current firstline treatments. ${ }^{4}$ Given this, there is clear scope for the development of new, effective interventions that are more accessible, are less resource-intensive and target known cognitive mechanisms for the alleviation of symptoms.

One reliable cognitive marker in depression is an impairment in the ability to recall detailed autobiographical memories. ${ }^{5}$ Specifically, depressed individuals, relative to non-depressed, more often fail to recall memories of event-specific experiences that occurred within the space of a day, and instead provide memories of experiences that extended over longer periods of time, categories of repeated events, or abstractions of various experiences. ${ }^{5}{ }^{6}$ This occurs independent of whether the memory is positive, negative or neutrally valenced. This impairment, termed overgeneral memory or reduced autobiographical memory specificity, is recognised as a factor in the onset and maintenance of clinical depression. ${ }^{7}$ This is likely due to the critical role that retrieving detailed information from specific past experiences plays in healthy psychological functioning. For example, an impaired ability to retrieve specific autobiographical memory (AM) is associated with poorer problem-solving and the ability to mentally simulate future events, ${ }^{8}$ which are central to imagining, 
planning and prediction. This, in turn, may lead to inaccurate appraisals of past events which then drives faulty decision-making and inferences. ${ }^{9}$ Without the ability to draw on our experiences in a detailed way, our capacity to successfully adapt in the world and maintain good mental health is compromised.

One cognitive intervention that has shown promise in remediating this cognitive deficit and alleviating depressive symptoms is memory specificity training (MeST) ${ }^{10}$ Simple in its conception and execution, the MeST intervention involves sustained practice in producing specific AM in response to a range of cue words. Over a series of weekly sessions (typically 4-5) in a group-based format, facilitators prompt participants to self-generate specific AM to cue words. They then provide feedback on whether these responses are specific or not, and encourage more detailed and elaborated AM retrieval. To date, findings from studies of MeST for clinical depression have been promising. An initial pilot trial of MeST with inpatients indicated an increase in memory specificity following four weekly group-based sessions. ${ }^{10}$ A subsequent, randomised controlled trial again showed significant increases in memory specificity and subsequent significant reductions in depressive symptoms. ${ }^{11}$ An uncontrolled trial of MeST with a sample of depressed individuals on an outpatient wait-list also found significant increases in memory specificity and decreases in depressive symptoms. ${ }^{12}$ Finally, a recent cluster-randomised trial demonstrated that MeST leads to improvements in memory specificity, and was equivalent with another effective intervention (psychoeducation and supportive counselling) in producing large reductions in depressive symptoms. ${ }^{13}{ }^{14}$ Overall then, evidence indicates that the face-to-face MeST training programme can produce significant improvements in AM specificity and reductions in depressive symptoms.

Given the straightforward nature of MeST, it may be a good candidate for adaptation to an online delivery format. Online interventions for depression can be clinically effective ${ }^{15}$, as well as cost-effective, ${ }^{16}$ and provide an accessible, less resource-intensive option for sufferers that can be widely disseminated. Recently, a fully automated, online, computerised version of the MeST training programme (c-MeST) has demonstrated the ability to increase AM specificity in a non-clinical sample. ${ }^{17}$ Consistent with face-to-face MeST, participants provide written responses to cue words over a series of sessions. They receive feedback as to whether AMs are specific or not according to a computerised algorithm which is trained to distinguish these differences using machine learning. ${ }^{18} 19$ Given that c-MeST has been shown to improve memory specificity, the next step is to test this adaptation in a clinical sample to assess whether it can also produce reductions in depressive symptoms. Significantly, participants engaging in the online c-MeST programme will not be exposed to interactions with a facilitator or other group members. This will help to mitigate against the confounding effect of common therapy and interpersonal factors with any specific effect of increasing AM specificity on depressive symptoms, and therefore isolate this mechanism of change.

The aim of this study will be to conduct the first randomised controlled trial of an online adaptation of MeST in a sample of individuals with a current major depressive episode (MDE). Given the experimental nature of online MeST in a clinical group, a no-treatment comparator group was chosen against which to test for treatment efficacy. It is hypothesised that individuals that engage in c-MeST, relative to a control group, will report a lower incidence of MDE and significantly lower levels of depressive symptoms at postintervention and follow-up time points. It is also hypothesised that changes in AM specificity between preintervention and postintervention will predict changes in depressive symptoms.

\section{METHODS AND ANALYSIS}

\section{Study design}

To test the study hypotheses, we will conduct an online, randomised controlled trial, using a 2 (condition: c-MeST, no c-MeST control group) x 4 (time point: baseline, postintervention, 1 and 3 months postintervention) factorial design. The control group will complete assessments and be offered access to the intervention at the end of the study. Although MeST has demonstrated efficacy in reducing depressive symptoms in a face-to-face format, this has not been established in an online format. Therefore, this phase II trial will determine whether c-MeST is superior to a wait-list control group, and estimate effect sizes for a larger trial comparing it to other interventions or as an adjunct intervention.

\section{Participants and recruitment}

The inclusion criteria, assessed through self-report, will be: (1) $\geq 18$ years of age, (2) residing in Australia, (3) fluent in English, (4) a current diagnosis of MDE as assessed by Diagnostic and Statistical Manual for Mental Disorders, fifth edition (DSM-5) criteria ${ }^{20}$ using the automated electronic psychological assessment system (e-PASS), ${ }^{21}$ (5) a score on the Patient Health Questionnaire (PHQ) of at least $10^{22}$ and (6) internet access in the home. The exclusion criteria, also assessed through self-report, will be (2) currently receiving any treatment for depression, with the exception of antidepressant medication that has been stable in type and dosage for at least 4 weeks, and (2) PHQ scores of $\geq 21$, indicative of highly severe depressive symptoms. Therefore, the sample will include those with moderate and severe symptoms, but will exclude those who are highly severe, due to the lack of provision for assertive follow-up for those that are highly unwell.

Randomisation will be conducted by a computerised algorithm using a 1:1 ratio, and will occur following informed consent and completion of the baseline questionnaire.

Participants will be recruited via advertisements posted in a range of formats, such as online advertisements (eg, on depression-related internet forums in Australia and on 


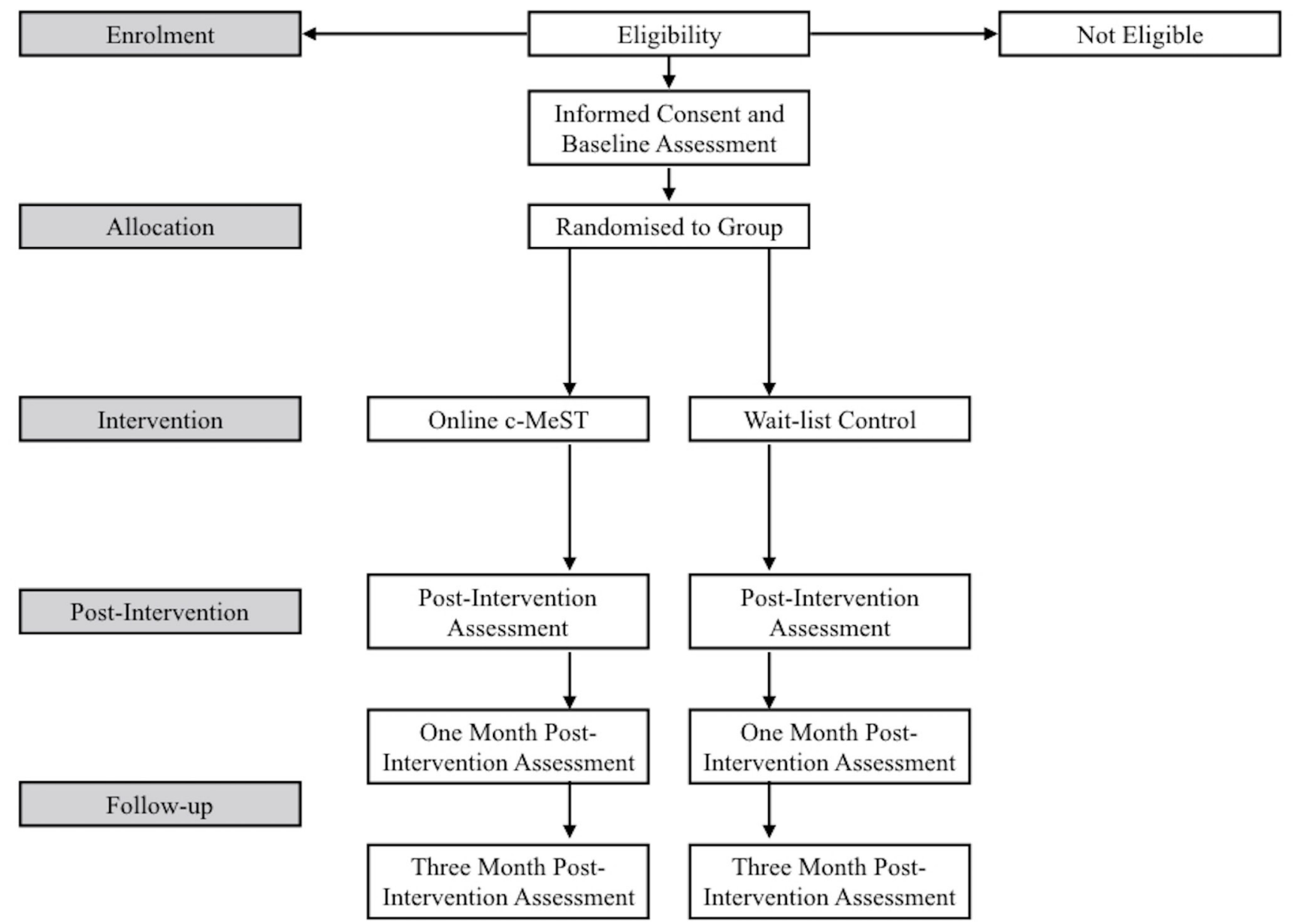

Figure 1 Study design for participant flow through the trial. MeST, memory specificity training.

Facebook), flyers sent to community groups and media releases. Those who wish to take part in the study will follow provided links to an online portal where they will be presented with information about the study and the plain language statement. After reading this, it will be clearly indicated that clicking continue onto the screening questions to assess eligibility will imply that informed consent has been given. Should they be eligible for the study, they will complete the baseline questionnaire and be randomised to one of the two conditions. Continued use of c-MeST and completion of the postintervention and follow-up questionnaires will imply ongoing consent. Figure 1 shows the intended participant flow.

\section{Online c-MeST intervention}

The c-MeST programme will comprise seven sessions based on content from validated methods of improving AM specificity in face-to-face ${ }^{10}$ and online formats. ${ }^{11}$ Initially, the aims, rationale, structure and process of the intervention will be briefly presented to participants. In each session, participants will be provided with a series of positive, negative and neutral cue words and are asked to provide a memory of an event prompted by the word that is specific in nature. The bank of cue words is selected from a word database and balanced for frequency, emotional valence and arousal ${ }^{23}$ and will be presented randomly across the sessions. In each session, participants will be asked to provide a memory of an event that occurred yesterday and on that day. Participants will be provided with automated feedback as to whether the response is specific or not via the application of an algorithm developed to classify written autobiographical memories. ${ }^{18}$ Where responses are not specific, participants will be asked to reattempt, and will be given feedback to add episodic detail to the response (eg, when, where, and with whom the experience occurred), and ensure it is discussed as past tense and is specific in nature rather than an extended experience that lasts more than a day, or a category of events. Participants will then be given another two opportunities to provide a specific response before the next cue word is displayed. Once they have received feedback that the response is specific, they will be prompted to add more episodic detail for the event. In sessions 1 to 3 , participants will be asked to provide one response to cue words and episodic details of those memories. In sessions 4 to 7 , participants will provide two different responses to a single cue word in order to improve their ability to identify what characterises specific instances of experience. The participants will be able to 
complete the modules at their convenience over a 2-week period, but will be advised not to do multiple sessions in 1 day.

\section{Primary outcomes}

The primary outcome measures will be online assessment of changes in MDE diagnostic status and level of self-reported depressive symptoms at postintervention. One-month and three-month follow-ups will also be conducted. The e-PASS,${ }^{21}$ a fully automated online self-report diagnostic system, will be used to assess MDE as consistent with DSM- 5 criteria. ${ }^{20}$ The e-PASS has demonstrated good concordance with 'gold-standard' structured, clinical interviews. ${ }^{21} \mathrm{PHQ}^{22}$ will be used to assess self-reported depressive symptoms. PHQ is a nine-item self-report scale, with items corresponding to the DSM-5 criteria for an MDE. ${ }^{20}$ Respondents rate themselves on each criterion from 0 (not at all) to 3 (nearly every day) to indicate how often they have experienced the symptoms in the last 2 weeks. We chose an inclusion criterion of at least 10 points on the PHQ indicative of at least moderate severity of symptoms. This cut-off has demonstrated high specificity and sensitivity in detecting cases of MDE. ${ }^{24}$ The PHQ has demonstrated excellent validity and reliability across numerous studies. ${ }^{25}$

\section{Secondary outcomes}

For secondary outcomes, all completed online, we will assess changes in the mean number of responses determined to be 'specific' on the Autobiographical Memory Test $^{26}$ and the Episodic Future Thinking Test ${ }^{27}$ (ie, past or future experiences, respectively, that are coded as occurring within 24 hours). This latter assessment will be of an exploratory nature, and in the context of episodic future thinking specificity being strongly, if not intrinsically, related to AM, and also known to be impaired in the context of clinical depression. ${ }^{8}$ We will also assess changes in mean scores for variables thought to be related to change in AM specificity: rumination as assessed by the Ruminative Responses Scale, ${ }^{28}$ cognitive avoidance as assessed by the White Bear Suppression Inventory ${ }^{29}$ and verbal fluency as assessed by the Controlled Oral Word Association Test. ${ }^{30}$ Additional outcomes of interest assessed through mean score change will be suicidal ideation as assessed by the Suicidal Ideation Attributes Scale, ${ }^{31}$ symptoms of clinical anxiety as assessed by the Generalised Anxiety Disorder Scale,$^{32}$ self-efficacy as assessed by the New General Self-Efficacy Scale ${ }^{33}$ and effective problem-solving as assessed by the Means-End Problem Solving Procedure. ${ }^{34}$ Parallel forms of the Autobiographical Memory Test, Episodic Future Thinking-Test, Controlled Oral Word Association Test and Means-End Problem Solving Procedure will be counterbalanced across the time points. All measures will be assessed at all time points, from baseline to 3-month follow-up, with the exception of the Episodic Future Thinking-Test which will be administered only at preintervention and postintervention, and the Means-End Problem Solving Procedure which will be administered at preintervention and postintervention and 3-month follow-up due to availability of parallel versions.

\section{Power calculation}

Using $G^{*}$ Power V.3.1, ${ }^{35}$ it was calculated that a total sample of 105 participants will be needed. This will account for an attrition rate of $35 \%$ between baseline and 3-month follow-up. This remaining sample of 68 will provide $80 \%$ statistical power to detect a moderate $(d=0.60)$ group by time point interaction effect at postintervention and follow-up time points with a 5\% type I error rate, and a two-sided tail. This effect is conservative relative to previous findings of MeST outcomes relative to a control group. $^{11}$

\section{Statistical analysis}

All data will be stored online on a secure server, congruent with all assessment being completed in an online format. Only the researchers will have access to these data. Data will be downloaded from this server prior to analyses. The data analyst will be blinded to the condition of participants. Analyses will be on an intention-to-treat basis, with supplementary per protocol analyses. Linear mixed models will be conducted to assess differences between conditions on the primary and secondary outcomes at each time point. This statistical method will facilitate inclusion of participants with missing data. Condition, time point, and their interaction, will be modelled as fixed effects. Participants will be modelled as random effects at time points. Should there be differences between the groups in other treatment sought during their participation in the trial, this will be used as a covariate in analysis. To test mediation effects, primarily whether changes in AMT scores from baseline postintervention predict changes in depressive symptoms from postintervention to follow-ups, analyses will be conducted using a bias-corrected bootstrap test. Power calculations with correlations from the literature indicate that the sample size will provide power $(\beta=0.80, \alpha=0.05$, two-tailed test) to detect associations of a moderate effect size.

\section{Procedure}

After being identified as eligible for the study and providing informed consent, participants will be randomised. The researchers will be blinded to this process. After completion of the baseline assessments, participants randomised to the c-MeST group will be assigned a unique identifier code with which to access the online training programme. These participants will have 2 weeks in which to access the c-MeST programme, at which point their unique identifier code will no longer allow access. They will be asked to complete another set of outcome measures at this point, and then again 1 and 3 months later. During the 2 weeks of access to the training, they will receive daily email prompts with links to encourage them to access and complete the programme. Participants in the control group will also be prompted to complete the outcome measures at all time points. At the cessation of data 
collection, participants in the control group will be given access to the training programme until such time the data analysis is complete and the study findings are concluded.

\section{Patient and public involvement}

The research question and design of the MeST programme were informed by qualitative and quantitative participant feedback from two previous studies using online MeST. Participants will not be directly involved in the recruitment or conducting of the study. Results will be available to study participants in aggregated format following the projects completion.

\section{Ethics and dissemination}

Individuals interested in participating will provide informed consent prior to commencing their involvement. They will have the opportunity to contact one of the researchers to raise any questions or concerns they might have, and this is encouraged in the plain language form that is provided. They can withdraw from the study at any point. Regarding research governance, the study has received ethics clearance, and will be conducted in accordance with the Australian-based National Health and Medical Research Council's National Statement on Ethical Conduct in Human Research (2007). Any amendments made to this protocol prior to or during recruitment will be published on the Australian New Zealand Clinical Trials Registry. The study will also be conducted in compliance with the university guidelines and policies, and the university will monitor the study through regular reporting. Any harms or adverse events that are identified will be reported to them as they occur. The results of the study will be disseminated through publication at peer-reviewed scientific journals, and through presentations of the findings at conferences and workshops. With respect to safety, and in particular risk of harm to self, information regarding support services will be provided to all participants at each time point. Where participants score higher than a cut-off on the Suicidal Ideation Attributes Scale $^{32}$ indicative of increased risk for suicidal behaviour, they will be contacted via email, provided with information on support services and encouraged to access them at that time. Ongoing involvement in the study will be at their discretion.

\section{Trial registration}

The trial was registered on 8 September 2017 with the Australian New Zealand Clinical Trials Registry (identifier ACTRN12618000257268). Recruitment commenced on 25 August 2018. Recruitment is planned to cease in the first quarter of 2019.

\section{DISCUSSION}

As the demand for mental health interventions increases, so, too, will the impetus for easily deliverable and cost-effective treatments for depression. Training interventions that target disruptions and impairments in autobiographical memory represent one form of low-intensity treatment for depressive symptoms that have shown promise. ${ }^{36} \mathrm{In}$ particular, the evidence base for MeST, and the recent development of an automated online version, indicates its utility for this purpose. This study will evaluate the efficacy of a fully automated c-MeST programme in a sample of adults currently experiencing MDE. As noted, this will provide a test of whether training in the retrieval of specific autobiographical memories can produce reductions in depressive symptoms when divorced from other factors likely to be therapeutic within a face-to-face group setting. If efficacious, it will provide evidence for an intervention that is highly accessible and needs very few resources, making it suitable for broader rollout. The study will also provide insight into whether c-MeST will affect variables thought to be related to difficulties in retrieving specific AM (namely, rumination, cognitive avoidance and poorer executive functioning). ${ }^{37}$ It will also assess other outcomes that may indicate a broader application of c-MeST, such as improving episodic future thinking in depression. Should c-MeST be efficacious, then the findings of this trial will help to inform future studies, such as comparing c-MeST with another effective treatment, or assessing c-MeST as an adjunct treatment for depression.

Contributors DJH designed the study with input from DA, KT and FR. FR developed the MeST intervention materials, KT and FR developed the online adaptation, c-MeST. DJH drafted the study protocol manuscript and all authors contributed to redrafting.

Funding The lead author received support for this research from Deakin University, as part of a Deakin Health Postdoctoral Research Fellowship.

Competing interests None declared.

Patient consent for publication Not required.

Ethics approval DeakinUniversity Human Research Ethics Committee, reference number: 2017_168.

Provenance and peer review Not commissioned; externally peer reviewed.

Open access This is an open access article distributed in accordance with the Creative Commons Attribution Non Commercial (CC BY-NC 4.0) license, which permits others to distribute, remix, adapt, build upon this work non-commercially, and license their derivative works on different terms, provided the original work is properly cited, appropriate credit is given, any changes made indicated, and the use is non-commercial. See: http://creativecommons.org/licenses/by-nc/4.0/.

\section{REFERENCES}

1. Depression and other common mental disorders: Global health estimates. Report. Geneva: World Health Organisation, 2017.

2. Burgess PM, Pirkis JE, Slade TN, et al. Service use for mental health problems: findings from the 2007 national survey of mental health and wellbeing. Aust N Z J Psychiatry 2009;43:615-23.

3. Parslow RA, Jorm AF. Who uses mental health services in Australia? An analysis of data from the National Survey of Mental Health and Wellbeing. Aust N Z J Psychiatry 2000;34:997-1008.

4. Warden D, Rush AJ, Trivedi MH, et al. The STAR*D Project results: a comprehensive review of findings. Curr Psychiatry Rep 2007;9:449-59.

5. Williams JM, Barnhofer T, Crane C, et al. Autobiographical memory specificity and emotional disorder. Psychol Bull 2007;133:122-48.

6. Liu X, Li L, Xiao J, et al. Abnormalities of autobiographical memory of patients with depressive disorders: A meta-analysis. Psychol Psychother 2013;86:353-73.

7. Sumner JA, Griffith JW, Mineka S. Overgeneral autobiographical memory as a predictor of the course of depression: a meta-analysis. Behav Res Ther 2010;48:614-25. 
8. Hallford DJ, Austin DW, Takano K, et al. Psychopathology and episodic future thinking: A systematic review and meta-analysis of specificity and episodic detail. Behav Res Ther 2018;102:42-51.

9. Dalgleish T, Werner-Seidler A. Disruptions in autobiographical memory processing in depression and the emergence of memory therapeutics. Trends Cogn Sci 2014;18:596-604.

10. Raes F, Williams JM, Hermans D. Reducing cognitive vulnerability to depression: a preliminary investigation of MEmory Specificity Training (MEST) in inpatients with depressive symptomatology. J Behav Ther Exp Psychiatry 2009;40:24-38.

11. Neshat-Doost HT, Dalgleish T, Yule W, et al. Enhancing autobiographical memory specificity through cognitive training: An intervention for depression translated from basic science. Clin Psychol Sci 2012;1:84-92.

12. Eigenhuis E, Seldenrijk $A$, van Schaik $A$, et al. Feasibility and effectiveness of memory specificity training in depressed outpatients: A pilot study. Clin Psychol Psychother 2017;24:269-77.

13. Dalgleish T, Bevan A, McKinnon A, et al. A comparison of MEmory Specificity Training (MEST) to education and support (ES) in the treatment of recurrent depression: study protocol for a cluster randomised controlled trial. Trials 2014;15:293.

14. Werner-Seidler A, Hitchcock C, Bevan A, et al. A cluster randomized controlled platform trial comparing group MEmory specificity training (MEST) to group psychoeducation and supportive counselling (PSC) in the treatment of recurrent depression. Behav Res Ther 2018:105:1-9.

15. Karyotaki E, Riper $\mathrm{H}$, Twisk J, et al. Efficacy of self-guided internetbased cognitive behavioral therapy in the treatment of depressive symptoms: A meta-analysis of individual participant data. JAMA Psychiatry 2017;74:351-9.

16. Donker T, Blankers M, Hedman E, et al. Economic evaluations of Internet interventions for mental health: a systematic review. Psychol Med 2015;45:3357-76.

17. Takano K, Moriya J, Raes F. lost in distractors: reduced autobiographical memory specificity and dispersed activation spreading over distractors in working memory. Behav Res Ther 2017:94:19-35.

18. Takano K, Hallford DJ, Vanderveren E, et al. The computerized scoring algorithm for the autobiographical memory test: updates and extensions for analyzing memories of English-speaking adults. Memory 2018;6:1-8.

19. Takano K, Ueno M, Moriya J, et al. Unraveling the linguistic nature of specific autobiographical memories using a computerized classification algorithm. Behav Res Methods 2017;49:835-52.

20. American Psychiatric Association. Diagnostic and Statistical Manual of Mental Disorders. 5th ed. VA: ArlingtonAmerican Psychiatric Publishing, 2013.

21. Nguyen DP, Klein B, Meyer D, et al. The diagnostic validity and reliability of an internet-based clinical assessment program for mental disorders. J Med Internet Res 2015;17:e218.
22. Spitzer RL, Kroenke K, Williams JB, et al. Validation and utility of a self-report version of PRIME-MD: the PHQ primary care study. JAMA 1999;282:1737-44.

23. Bradley MM, Lang PJ. Affective norms for English words (ANEW): Instruction manual and affective ratings. Technical Report C-1, The Center for Research in Psychophysiology: University of Florida, 1999.

24. Manea L, Gilbody S, McMillan D. Optimal cut-off score for diagnosing depression with the Patient Health Questionnaire (PHQ9): a meta-analysis. CMAJ 2012;184:E191-E196.

25. Kroenke K, Spitzer RL, Williams JB, et al. The patient health questionnaire somatic, anxiety, and depressive symptom scales: A systematic review. Gen Hosp Psychiatry 2010;32:345-59.

26. Williams JM, Broadbent K. Autobiographical memory in suicide attempters. J Abnorm Psychol 1986;95:144-9.

27. Hallford DJ, Austin DW, Takano K, et al. Psychometric Evaluation of an Episodic Future Thinking Variant of the Autobiographical Memory Test (Episodic Future Thinking - Test; EFT-T). Under Review 2018.

28. Treynor W, Gonzalez R, Nolen-Hoeksema S. Rumination reconsidered: a psychometric analysis. Cognit Ther Res 2003;27:247-59.

29. Wegner DM, Zanakos S. Chronic thought suppression. J Pers 1994;62:615-40.

30. Ruff RM, Light RH, Parker SB, et al. BEnton controlled oral word association test: Reliability and updated norms. Arch Clin Neuropsychol 1996;11:329-38.

31. van Spijker BA, Batterham PJ, Calear AL, et al. The suicidal ideation attributes scale (SIDAS): Community-based validation study of a new scale for the measurement of suicidal ideation. Suicide Life Threat Behav 2014;44:408-19.

32. Spitzer RL, Kroenke K, Williams JB, et al. A brief measure for assessing generalized anxiety disorder: the GAD-7. Arch Intern Med 2006;166:1092-7.

33. Chen G, Gully SM, Eden D. Validation of a new general self-efficacy scale. Organ Res Methods 2001;4:62-83.

34. Platt JJ, Spivak G, Bloom MR, et al. MEPS): Manual and tentative norms. Research and Evaluation Report No. 29, Hahnemann; Medical College and Hospital, Department of Mental Health Sciences 1971

35. Faul $F$, Erdfelder E, Lang A-G, et al. G*Power 3: A flexible statistical power analysis program for the social, behavioral, and biomedical sciences. Behav Res Methods 2007;39:175-91.

36. Hitchcock C, Werner-Seidler A, Blackwell SE, et al. Autobiographical episodic memory-based training for the treatment of mood, anxiety and stress-related disorders: A systematic review and meta-analysis. Clin Psychol Rev 2017;52:92-107.

37. Williams JM. Capture and rumination, functional avoidance, and executive control (CaRFAX): Three processes that underlie overgeneral memory. Cogn Emot 2006;20(3-4):548-68. 